\title{
A potent risk model for predicting new-onset acute coronary syndrome in patients with type 2 diabetes mellitus in Northwest China
}

\author{
Jun Lyu ${ }^{1} \cdot$ Zhiying Li $^{2} \cdot$ Huiyi Wei ${ }^{3} \cdot$ Dandan $\mathrm{Liu}^{2} \cdot{\text { Xiaoxian } \mathrm{Chi}^{2} \cdot \text { Da-Wei Gong }}^{4} \cdot$ Qingbin Zhao $^{2} \odot$
}

Received: 27 October 2019 / Accepted: 14 January 2020 / Published online: 1 February 2020

(c) The Author(s) 2020

\begin{abstract}
Aims Type 2 diabetes mellitus (T2DM) is now very prevalent in China. Due to the lower rate of controlled diabetes in China compared to that in developed countries, there is a higher incidence of serious cardiovascular complications, especially acute coronary syndrome (ACS). The aim of this study was to establish a potent risk predictive model in the economically disadvantaged northwest region of China, which could predict the probability of new-onset ACS in patients with T2DM. Methods Of 456 patients with T2DM admitted to the First Affiliated Hospital of Xi' an Jiaotong University from January 2018 to January 2019 and included in this study, 270 had no ACS, while 186 had newly diagnosed ACS. Overall, 32 demographic characteristics and serum biomarkers of the study patients were analysed. The least absolute shrinkage and selection operator regression was used to select variables, while the multivariate logistic regression was used to establish the predictive model that was presented using a nomogram. The area under the receiver operating characteristics curve (AUC) was used to evaluate the discriminatory capacity of the model. A calibration plot and Hosmer-Lemeshow test were used for the calibration of the predictive model, while the decision curve analysis (DCA) was used to evaluate its clinical validity.

Results After random sampling, 319 and 137 T2DM patients were included in the training and validation sets, respectively. The predictive model included age, body mass index, diabetes duration, systolic blood pressure (SBP), diastolic blood pressure (DBP), low-density lipoprotein cholesterol, serum uric acid, lipoprotein(a), hypertension history and alcohol drinking status as predictors. The AUC of the predictive model and that of the internal validation set was 0.830 [95\% confidence interval (CI) 0.786-0.874] and 0.827 (95\% CI 0.756-0.899), respectively. The predictive model showed very good fitting degree, and DCA demonstrated a clinically effective predictive model.

Conclusions A potent risk predictive model was established, which is of great value for the secondary prevention of diabetes. Weight loss, lowering of SBP and blood uric acid levels and appropriate control for DBP may significantly reduce the risk of new-onset ACS in T2DM patients in Northwest China.
\end{abstract}

Keywords Cardiovascular disease $\cdot$ Type 2 diabetes mellitus $\cdot$ Risk predictive model $\cdot$ Northwest China

Managed by Massimo Federici.

Qingbin Zhao

zhaoqingbin05@163.com

1 Clinical Research Center, The First Affiliated Hospital of Xi' an Jiaotong University, Xi' an 710061, Shaanxi, China

2 Department of Geratology, The First Affiliated Hospital of Xi' an Jiaotong University, Xi' an 710061, Shaanxi, China

3 The Second Affiliated Middle School of Xi' an Jiaotong University, Xi' an 710061, Shaanxi, China

4 Division of Endocrinology, Diabetes and Nutrition, Department of Medicine, University of Maryland School of Medicine, Baltimore 21201, USA

$\begin{array}{ll}\text { Abbreviations } \\ \text { ACS } & \text { Acute coronary syndrome } \\ \text { AUC } & \text { Area under the curve } \\ \text { BMI } & \text { Body mass index } \\ \text { CHD } & \text { Coronary heart disease } \\ \text { CI } & \text { Confidence interval } \\ \text { CVD } & \text { Cardiovascular disease } \\ \text { DBP } & \text { Diastolic blood pressure } \\ \text { DCA } & \text { Decision curve analysis } \\ \text { LASSO } & \text { The least absolute shrinkage and selection } \\ & \text { operator } \\ \text { LDL-C } & \text { Low-density lipoprotein cholesterol } \\ \text { Lp(a) } & \text { Lipoprotein(a) } \\ \text { OR } & \text { Odds ratio } \\ \text { PCIs } & \text { Percutaneous coronary interventions }\end{array}$


ROC Receiver operating characteristics

SBP Systolic blood pressure

SUA Serum uric acid

T2DM Type 2 diabetes mellitus

\section{Background}

At present, diabetes has become an epidemic in developing countries, posing a serious threat to people's health with increasing medical burden. The prevalence of type 2 diabetes mellitus (T2DM) in Chinese adults (2018) was $12.75 \%$. Diabetes is an independent risk factor for cardiovascular disease (CVD). Compared with non-diabetic population, the risk of CVD in diabetic patients is $2-4$ times higher [1-3]. Having diabetes is often associated with important risk factors of CVD such as hypertension and dyslipidaemia [4]. Clinical evidence shows that strict blood glucose control offers a limited effect in reducing the risk of CVD and related deaths in patients with T2DM, especially those with a longer course of disease, the older age, those with previous CVD, or with multiple cardiovascular risk factors [5]. However, comprehensive control of multiple risk factors can markedly decrease the risk of CVD and deaths in patients with diabetes [6]. Currently, in Chinese T2DM patients, the incidence of cardiovascular risk factors is high, but the control rate is low. In patients with T2DM who are treated in the outpatient clinics, the comprehensive compliance rate of blood glucose, blood pressure and blood lipid controlling was only 5.6\% [4].

Acute coronary syndrome (ACS) is the most serious clinical phenotype of CVD. Although there are many treatments for ACS, the mortality, recurrent myocardial infarction and repeated hospitalization of ACS patients remain high. Most of the hospitalized ACS patients have dysglycaemia [7]. In clinical practice in the low-income Northwest China, diabetes is very commonly found in ACS patients in the intensive coronary care unit. In this setting, diabetes is often complicated with coronary multi-vessel diseases, often requiring multiple percutaneous coronary interventions (PCIs), with a high incidence of repeated hospitalization, heart failure and poor prognosis.

Due to the high incidence of T2DM in low-income areas in China, there are high mortality and heavy medical burden of ACS among diabetic patients. Thus, this study aimed to establish a potent risk predictive model, which can provide early prediction of the risk of new-onset ACS in T2DM patients, thereby reducing the adverse cardiovascular outcomes in such patients in the region.

\section{Methods}

\section{Study design and setting}

This study collected data from 456 T2DM patients who were admitted to the First Affiliated Hospital of Xi' an Jiaotong University from January 2018 to January 2019. The ethics committee of the medical centre approved the study (Reference Number: XJTU1AF2019LSK-064).

Patient demographic characteristics, serum biomarkers and coronary angiography data were collected by trained researchers at the study centre. All data were then submitted electronically to a central department for verification. All experiments were approved by the First Affiliated Hospital of Xi' an Jiaotong University, and all methods were carried out in accordance with international guidelines and regulations. A written informed consent was obtained from all participants.

\section{Study participants}

This study randomly selected 456 T2DM patients hospitalized in the medical centre from January 2018 to January 2019. Of these, 186 had both T2DM and ACS and were newly diagnosed either with ST-segment elevation myocardial infarction or non-ST-segment elevation myocardial infarction, or unstable angina, according to the recommended criteria by the Joint European Society of Cardiology and the American College of Cardiology Committee [8]. The remaining 270 patients had T2DM without ACS. The diagnosis of T2DM was based on the World Health Organization and American Diabetes Association (ADA) criteria as follows: fasting plasma glucose concentration of $7.0 \mathrm{mmol} / \mathrm{L}(126 \mathrm{mg} / \mathrm{dL})$ or higher, or 2-h post-glucose load venous plasma glucose of $11.1 \mathrm{mmol} / \mathrm{L}(200 \mathrm{mg} / \mathrm{dL})$ or higher, confirmed on two occasions. The exclusion of type 1 diabetes mellitus was based on clinical distinction by the respective attending physicians, including young age of onset, positive islet autoantibody and history of insulindependent glycaemic control. Furthermore, gestational diabetes and other types of diabetes were also excluded.

All ACS patients underwent coronary angiography during the hospitalization, performed by at least two interventional doctors in the Department of Cardiology of the medical centre.

\section{Candidate predictors}

The collected demographic variables included age, sex, body mass index (BMI), smoking status, alcohol drinking status, hypertension history, T2DM duration, family history of 
coronary heart disease (CHD), systolic blood pressure (SBP) and diastolic blood pressure (DBP). BMI was calculated as weight $(\mathrm{kg}) / \mathrm{height}\left(\mathrm{m}^{2}\right)$, which were measured at admission.

Serum biomarkers included blood urea nitrogen, serum creatinine, cystatin C, serum uric acid (SUA), estimated glomerular filtration rate, total cholesterol, triglycerides, low-density lipoprotein cholesterol (LDL-C), high-density lipoprotein cholesterol (HDL-C), lipoprotein(a) [Lp(a)], apolipoprotein A (ApoA), apolipoprotein B (ApoB), apolipoprotein $\mathrm{E}$ (ApoE), platelet count, platelet distribution width, mean platelet volume, platelet-larger cell ratio, plateletcrit, haemoglobin A1c (HbAlc), fasting blood glucose, D-dimer and gamma-glutamyl transpeptidase.

\section{Statistical analysis}

The continuous variables with normal distribution were expressed as mean \pm standard deviation, while the categorical variables were expressed as percentages. To establish and validate the predictive model, $70 \%$ of the study patients were randomly selected as the training set and the other $30 \%$ as the validation set. The least absolute shrinkage and selection operator (LASSO) regression was used to select variables into the predictive model. By applying multivariate logistic regression, we established a predictive model. The risk predictive model of new-onset ACS in T2DM patients was presented using a nomogram. We evaluated the predictive model from three aspects, namely the discriminatory capacity, the calibration ability and the clinical effectiveness. The discriminatory capacity was evaluated by the area under the receiver operating characteristics (ROC) curve (AUC). The calibration ability was evaluated by a calibration plot and Hosmer-Lemeshow test. The clinical effectiveness was evaluated by the decision curve analysis (DCA).

All tests were two-tailed, and a $P$ value of $<0.05$ was considered statistically significant. All statistical analyses were performed using R software (version 3.5.1, Vienna, Austria).

\section{Results}

\section{Participant characteristics}

Following random sampling, 319 and 137 T2DM patients were included in the training and validation sets, respectively. The demographic and clinicobiochemical characteristics of the participants are listed in Table 1.

\section{Independent risk factors in the training set}

Multivariate logistic regression analysis demonstrated that age, BMI, diabetes mellitus duration, SBP, DBP, LDL-C, SUA, Lp(a), hypertension history and alcohol drinking status were independent risk factors for new-onset ACS in T2DM patients (Table 2).

\section{Predictive model construction}

The LASSO regression analysis was used to select variables from those shown in Table 1, and the multivariate logistic regression was used to establish the predictive model. The established predictive model included age, BMI, diabetes mellitus duration, SBP, DBP, LDL-C, SUA, Lp(a), hypertension history and alcohol drinking status as predictors. The predictive model was presented using a nomogram that was used to quantitatively predict the risk probability of newonset ACS in T2DM patients (Fig. 1).

\section{Weight of predictors}

From the nomogram of the predictive model, the single scores of age, BMI, SBP, DBP and SUA were all greater than 60 , indicating that these predictors had great weight in the model.

\section{Predictive model validation}

The AUC was used to evaluate the discriminatory capacity of the predictive model. For the predictive model, the AUC was 0.830 [95\% confidence interval (CI) 0.786-0.874], while that of the internal validation set was 0.827 (95\% CI 0.756-0.899) (Fig. 2).

A calibration plot and Hosmer-Lemeshow test were used for the calibration of the predictive model. From the calibration curves, the predictive model and the validation set showed very good fitting degree. As shown by the Hosmer-Lemeshow test, the predicted and actual probability was highly consistent (training set, $P=0.705$; validation set, $P=0.823$ ) (Fig. 3).

DCA was used to evaluate the clinical validity of the predictive model. From the decision curves, the net benefits of the predictive model and the internal validation set were significantly higher than those of the two extreme cases, that is, all people were treated (Fig. 4).

\section{Discussion}

For the first time, we established a risk prediction model with a strong predictive ability and an AUC that reached 0.830 . In addition, the predictive model established in this study had high calibration and clinical validity. According to the predictive model, the clinical characteristics of diabetic patients, such as age, BMI, diabetes duration, hypertension history, alcohol drinking status, SBP, DBP, LDL-C, SUA and $\mathrm{Lp}$ (a) levels, can be used as predictors. Therefore, in 
Table 1 Participant characteristics

\begin{tabular}{|c|c|c|c|}
\hline Variables & Training set $(n=319)$ & Validation set $(n=137)$ & $P$ value \\
\hline Status, $n(\%)$ & & & 0.153 \\
\hline $0=$ without ACS & $182(57.1)$ & $88(64.2)$ & \\
\hline $1=$ with ACS & $137(42.9)$ & $49(35.8)$ & \\
\hline Sex, $n(\%)$ & & & 0.64 \\
\hline $1=$ male & $219(68.7)$ & $91(66.4)$ & \\
\hline $2=$ female & $100(31.3)$ & $46(33.6)$ & \\
\hline Age, years & $56.7 \pm 10.2$ & $57.2 \pm 9.9$ & 0.591 \\
\hline T2DM duration, years & $8.6 \pm 5.7$ & $9.3 \pm 6.7$ & 0.244 \\
\hline Family history of CHD, $n(\%)$ & & & 0.141 \\
\hline $1=$ yes & $38(11.9)$ & $10(7.3)$ & \\
\hline $2=$ no & $281(88.1)$ & $127(92.7)$ & \\
\hline Hypertension, $n(\%)$ & & & 0.610 \\
\hline $1=$ yes & 155 (48.6) & $63(46.0)$ & \\
\hline $2=$ no & $164(51.4)$ & $74(54.0)$ & \\
\hline Drinking, $n(\%)$ & & & 0.013 \\
\hline $1=$ yes & 37 (11.6) & $28(20.4)$ & \\
\hline $2=$ no & $282(88.4)$ & 109 (79.6) & \\
\hline Smoking, $n(\%)$ & & & 0.879 \\
\hline $1=$ yes & $121(37.9)$ & $53(38.7)$ & \\
\hline $2=$ no & $198(62.1)$ & $84(61.3)$ & \\
\hline Body mass index, $\mathrm{kg} / \mathrm{m}^{2}$ & $25.8 \pm 3.7$ & $25.2 \pm 3.9$ & 0.131 \\
\hline Systolic blood pressure, $\mathrm{mmHg}$ & $132.7 \pm 18.1$ & $130.7 \pm 15.7$ & 0.262 \\
\hline Diastolic blood pressure, $\mathrm{mmHg}$ & $78.4 \pm 10.2$ & $76.8 \pm 9.7$ & 0.124 \\
\hline Haemoglobin Alc, $\%$ & $8.1 \pm 1.8$ & $8.1 \pm 1.8$ & 0.879 \\
\hline Fasting blood glucose, $\mathrm{mmol} / \mathrm{L}$ & $8.1 \pm 2.9$ & $7.7 \pm 2.6$ & 0.195 \\
\hline Total cholesterol, $\mathrm{mmol} / \mathrm{L}$ & $4.0 \pm 0.9$ & $4.1 \pm 1.0$ & 0.271 \\
\hline Triglyceride, $\mathrm{mmol} / \mathrm{L}$ & $1.9 \pm 1.6$ & $1.8 \pm 1.4$ & 0.623 \\
\hline High-density lipoprotein cholesterol, $\mathrm{mmol} / \mathrm{L}$ & $1.0 \pm 0.3$ & $1.0 \pm 0.3$ & 0.646 \\
\hline Low-density lipoprotein cholesterol, $\mathrm{mmol} / \mathrm{L}$ & $2.4 \pm 0.8$ & $2.5 \pm 0.8$ & 0.175 \\
\hline Apolipoprotein A, g/L & $1.1 \pm 0.2$ & $1.2 \pm 0.2$ & 0.342 \\
\hline Apolipoprotein B, g/L & $0.8 \pm 0.2$ & $0.8 \pm 0.2$ & 0.341 \\
\hline Apolipoprotein E, mg/L & $35.8 \pm 16.7$ & $35.6 \pm 16.5$ & 0.920 \\
\hline Lipoprotein(a), mg/L & $154.9 \pm 162.5$ & $153.8 \pm 169.9$ & 0.950 \\
\hline Estimated glomerular filtration rate, $\mathrm{ml} / \mathrm{min} / 1.73 \mathrm{~m}^{2}$ & $123.6 \pm 34.1$ & $124.9 \pm 30.4$ & 0.688 \\
\hline Blood urea nitrogen, mmol/L & $5.8 \pm 1.8$ & $5.7 \pm 1.6$ & 0.591 \\
\hline Serum creatinine, $\mathrm{mmol} / \mathrm{L}$ & $62.1 \pm 22.1$ & $59.5 \pm 15.4$ & 0.210 \\
\hline Cystatin C, mg/L & $0.8 \pm 0.3$ & $0.8 \pm 0.2$ & 0.309 \\
\hline Serum uric acid, $\mu \mathrm{mol} / \mathrm{L}$ & $334.2 \pm 80.1$ & $333.4 \pm 86.1$ & 0.920 \\
\hline D-dimer, mg/L & $0.5 \pm 0.5$ & $0.4 \pm 0.2$ & 0.419 \\
\hline Gamma-glutamyl transpeptidase, U/L & $31.1 \pm 24.0$ & $34.2 \pm 41.2$ & 0.320 \\
\hline Platelet count, $\times 109 / \mathrm{L}$ & $193.9 \pm 55.6$ & $189.5 \pm 52.7$ & 0.436 \\
\hline Platelet distribution width, $\mathrm{fL}$ & $15.5 \pm 3.0$ & $15.5 \pm 3.1$ & 0.917 \\
\hline Mean platelet volume, fL & $11.7 \pm 1.3$ & $11.7 \pm 1.3$ & 0.842 \\
\hline Platelet-larger cell ratio, $\%$ & $38.3 \pm 10.3$ & $38.8 \pm 10.1$ & 0.658 \\
\hline Plateletcrit, $\%$ & $0.2 \pm 0.1$ & $0.2 \pm 0.1$ & 0.285 \\
\hline
\end{tabular}

ACS, acute coronary syndrome; T2DM, type 2 diabetes mellitus; CHD, coronary heart disease 
Table 2 Multivariate logistic regression analysis (training set)

\begin{tabular}{lllr}
\hline Variables & OR & \multicolumn{1}{l}{$95 \%$ CI } & $P$ value \\
\hline Age & 1.067 & $1.033-1.103$ & $<0.001$ \\
Body mass index & 1.139 & $1.055-1.235$ & 0.001 \\
Diabetes duration & 0.943 & $0.893-0.992$ & 0.027 \\
Systolic blood pressure & 1.031 & $1.010-1.053$ & 0.005 \\
Diastolic blood pressure & 0.940 & $0.903-0.969$ & $<0.001$ \\
Low-density lipoprotein choles- & 0.547 & $0.371-0.792$ & 0.002 \\
$\quad$ terol & & & \\
Serum uric acid & 1.008 & $1.004-1.012$ & $<0.001$ \\
Lipoprotein(a) & 1.002 & $1.000-1.004$ & 0.028 \\
Hypertension & & & \\
$\quad$ 1=yes & Reference & & \\
$2=$ no & 0.417 & $0.232-0.741$ & 0.003 \\
Drinking & & & \\
$\quad$ 1=yes & Reference & & \\
$2=$ no & 1.497 & $0.621-3.864$ & 0.383 \\
\hline
\end{tabular}

OR, odds ratio; $\mathrm{CI}$, confidence interval

clinical practice, we can predict the risk probability of newonset ACS in T2DM patients by obtaining information about their general medical history, conducting physical examination and performing serum biochemical indicators testing. The predictive model can guide the formulation of ACS prevention strategies for T2DM in local areas.

Multiple cardiovascular risk factors exist in T2DM patients [9]. Even after adjusting for other factors, fourfold increased risk of heart failure was reported [10]. In 2015, the American Heart Association and the ADA (AHA/ADA) issued a statement [11], indicating that lifestyle, weight management, glycosylated haemoglobin level, hyperglycaemia or hypoglycaemia, hypertension, high cholesterol and hyperlipoproteinaemia are all risk factors for CVD in T2DM patients. At present, the prevalence of T2DM in China continues to rise, and the rate of patients with controlled T2DM is significantly lower than those in developed countries. Furthermore, many patients require PCI for ACS due to diabetes. These patients often show multiple vessel damage and poor prognosis, especially in Northwest China. This situation leads to serious shortage of medical resources. The population in Northwest China is characterized by lower educational and income level than that of Eastern China with a developed economy. The diet is characterized by high salt and oil intakes, and the staple food is mainly carbohydrate. There is an urgent need to establish an economical and effective risk prediction model for new-onset ACS in T2DM patients in this area. This model could provide answers to the factors that are related to the onset of ACS in T2DM patients in this population group; it could also predict the weight of each predictor in the risk prediction model. This will help to develop effective prevention strategies by clinicians in the region.

Previous studies have shown that age [12-16], hypertension [4, 17], obesity [18, 19], hyperuricaemia [20-24], dyslipidaemia [25] are all risk factors for CVD. Although the above results were obtained in the general population, these risk factors remain as predictors in the risk model established in this study. Currently, whether strict blood glucose control can reduce adverse cardiovascular outcomes in diabetic patients is still controversial. In patients with long course of T2DM, strict blood glucose management may not necessarily reduce adverse cardiovascular outcomes [26]. Studies have shown that hyperglycaemia is a relatively weak risk factor for CVD, and the anti-atherosclerosis effect of
Fig. 1 A nomogram for predicting the probability of new-onset ACS in T2DM patients. The nomogram is used by scoring each variable on its corresponding score scale. The scores for all variables are then summed up to obtain the total score, and a vertical line is drawn from the total point row to indicate the estimated probability of newonset ACS in T2DM patients. BMI, body mass index; SBP, systolic blood pressure; DBP, diastolic blood pressure; LDLC, low-density lipoprotein cholesterol; UA, uric acid; Lp(a), lipoprotein(a); Hp, hypertension history; ACS, acute coronary syndrome; and T2DM, type 2 diabetes mellitus

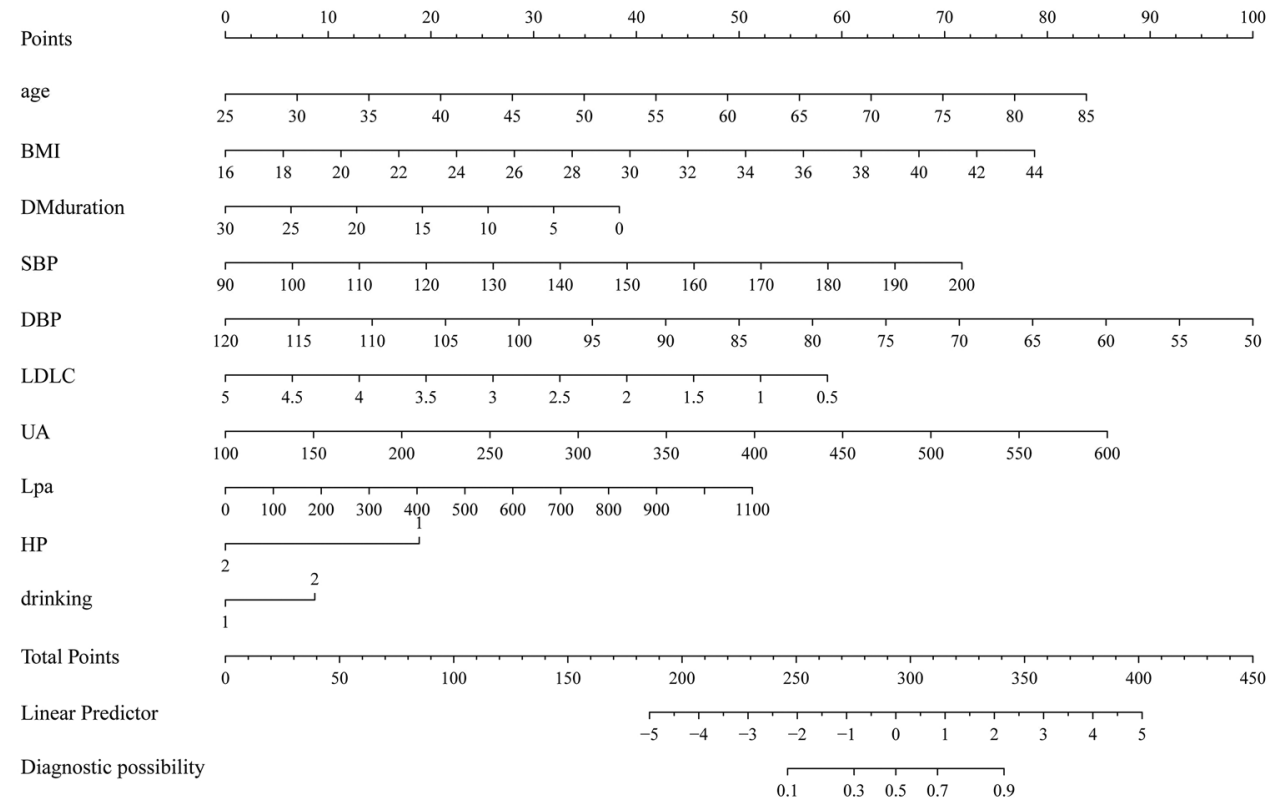



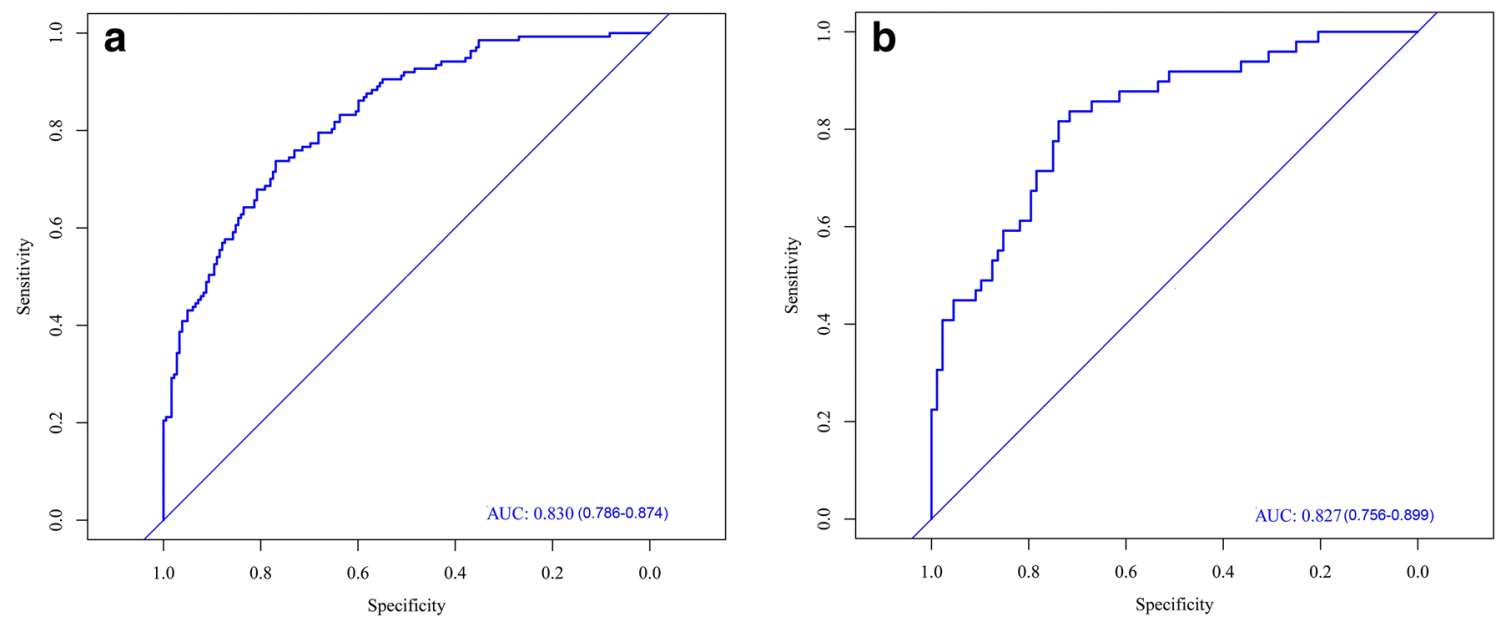

Fig. 2 ROC curves $\mathbf{a}$ from the training set and $\mathbf{b}$ from the validation set

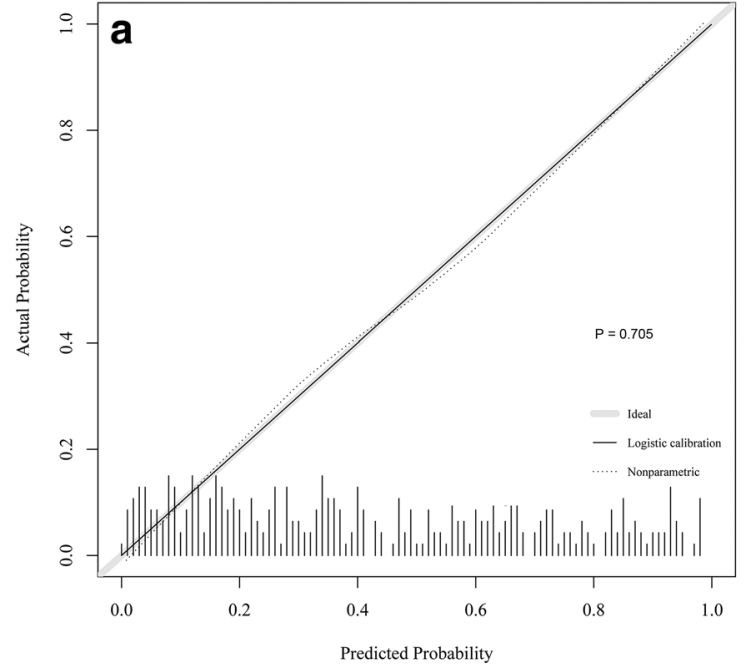

Fig. 3 Calibration plots. The shadow line represents a perfect prediction by an ideal model, and the dotted line shows the performance of the training set (a) and validation set (b). The Hosmer-Lemeshow

decreased HbA1c may take more than 10 years to show [27-29]. This present study did not find blood glucose level to be a predictor of ACS in T2DM. However, the risk of ACS in patients with long duration of T2DM was found to be low, but the weight of diabetes duration as a predictor was also low.

Studies have found that moderate drinking can reduce the incidence of cardiovascular events and total mortality in T2DM patients compared to non-drinking. Heavy drinking can increase the risk of cardiovascular events and total mortality in T2DM patients, and the risk and mortality of cardiovascular events in alcoholics showed a dose-response relationship with ethanol consumption [30]. This study found a lower risk of ACS in T2DM patients who drank

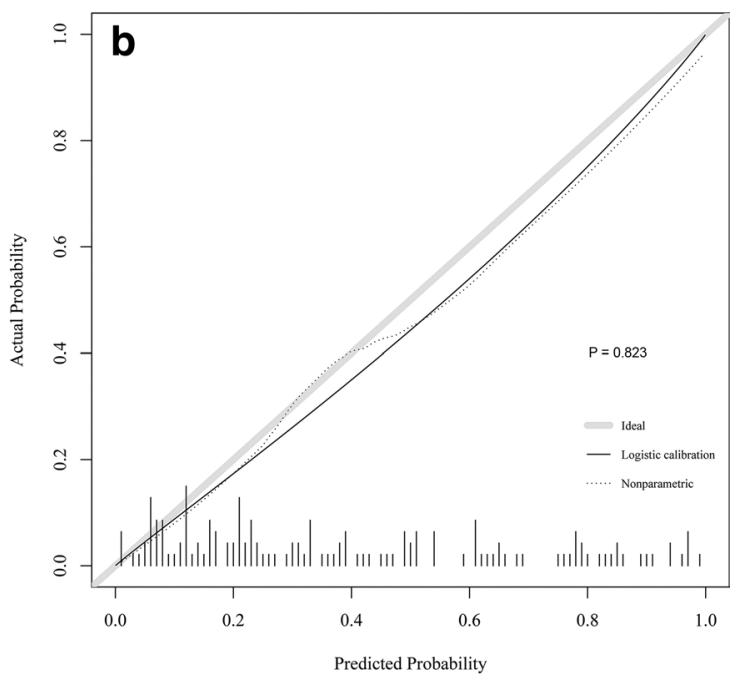

test yielded a $P$ value of 0.705 in the training set (a) and 0.823 in the validation set $(\mathbf{b})$

alcohol than that in non-alcoholic patients, but this study did not quantify alcohol consumption. There is evidence that in hypertensive individuals, aggressive lowering of the blood pressure through antihypertensive therapy may predispose to increased cardiovascular risk due to low DBP [31-33]. This study also found that the lower the DBP in T2DM patients, the higher the risk of ACS.

Elevated Lp(a) and LDL-C are associated with increased risk of CVD [34, 35]. There is an evolving body of evidence supporting the role of $\mathrm{Lp}(\mathrm{a})$ in the risk of coronary artery disease, in epidemiological studies [36, 37], and in Mendelian randomization [38] and genome-wide association studies [39]. A recent study demonstrated that LDL-C $\geq 190 \mathrm{mg} /$ $\mathrm{dL}(\geq 4.91 \mathrm{mmol} / \mathrm{L})$ is associated with an accelerated risk 


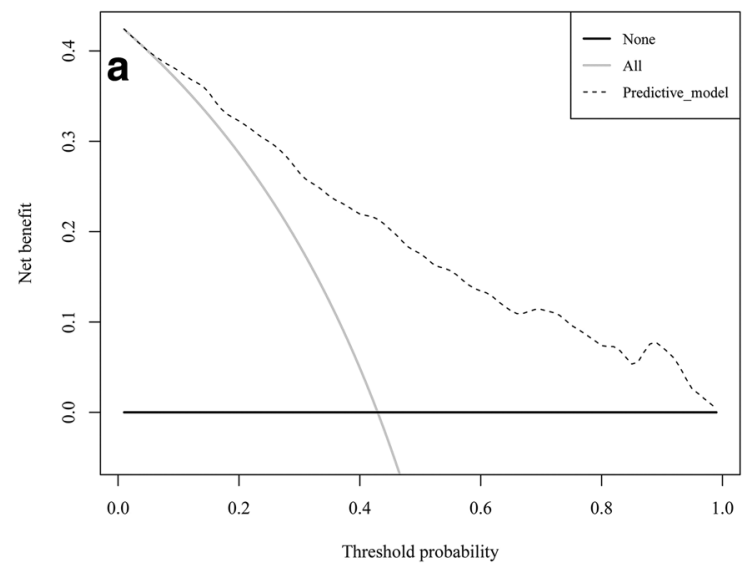

Fig. 4 Decision curve analysis. Area within the dotted line, the grey solid line, and the black solid line represents the net benefit. The black solid line indicates that all samples are negative and all were

of coronary artery disease [40] and the coexistence of both elevated LDL-C and Lp(a) may exacerbate this risk [41, 42]. This present study found that elevated $\mathrm{Lp}(\mathrm{a})$ increased the risk of ACS in T2DM, similar to previous studies in the general population. However, this study found that lower LDL-C levels increased the risk of ACS in T2DM, which is inconsistent with previous studies in the general population. We consider that diabetic patients at high risk of ACS in this region may have had elevated LDL-C in the past and taken statins. Recent studies demonstrated that the relationship between LDL-C and CVD depends on the level of LDL-C and the cumulative exposure time $[43,44]$.

Finally, our study also demonstrated that age, BMI, SBP, DBP and SUA played a large role in the predictive model. Therefore, in Northwest China, the risk of new-onset ACS in T2DM may be significantly reduced by lowering the body weight, SBP and blood uric acid levels and maintaining an appropriate DBP.

\section{Limitations}

There are some limitations in this study. Firstly, the prediction model established in this study suggests that alcohol drinking can reduce the risk of ACS in T2DM, but there is no detailed quantification of drinking, such as light or large drinking, which may lead to differences in results. Secondly, the prediction model suggests that low LDL-C level may increase the risk of ACS in T2DM. The data of patients in this study did not provide information about the use of lipid-regulating drugs, which might have been used by patients with low LDL-C at admission. Thirdly, although the sample size of this study was small, the AUC of the predictive model and that of the internal validation

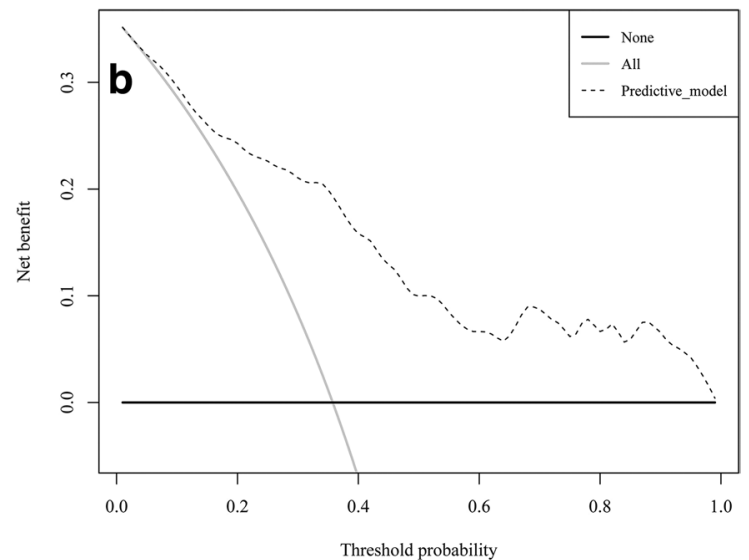

not treated. The grey solid line indicates that all samples were positive and were treated $\mathbf{a}$ from the training set and $\mathbf{b}$ from the validation set

set were 0.830 (95\%CI 0.786-0.874) and 0.827 (95\% CI 0.756-0.899), respectively. The predictive model showed very good fitting degree, and the DCA demonstrated a clinically effective predictive model. Finally, due to the large gap in the level of medical care in this region, a single-centre study was conducted to ensure the reliability of the study. Therefore, whether the results of this study can be extended to other regions and countries needs to be further verified by external cohorts.

\section{Conclusions}

In conclusion, we have established a cost-effective risk prediction model in Northwest China, and the application of this model may play an important role in preventing adverse cardiovascular outcomes in T2DM patients in this region.

Acknowledgements We would like to thank Editage (www.edita ge.com) for English language editing.

Author's contribution QBZ contributed to the conception and design. DDL, ZYL, XXC and HYW contributed to the data acquisition. JL and DWG contributed to the data analysis and interpretation. QBZ drafted the manuscript. All authors read and approved the final manuscript.

Funding This work was supported by Grants from Shaanxi Province Science and Technology Foundation of China (2019KW- 079) and National Natural Science Foundation of China (81970329).

Data availability The datasets used during the current study are available from the corresponding author on reasonable request. 


\section{Compliance with ethical standards}

Conflict of interest The authors declare that they have no competing interests.

Ethical standards The ethics committee of the First Affiliated Hospital of Xi' an Jiaotong University approved the study (Reference Number: XJTU1AF2019LSK-064). This study was performed in accordance with the ethical standards as laid down in the 1964 Declaration of Helsinki and its later amendments.

\section{Consent for publication Not applicable.}

Informed consent A written informed consent was obtained from all participants.

Open Access This article is licensed under a Creative Commons Attribution 4.0 International License, which permits use, sharing, adaptation, distribution and reproduction in any medium or format, as long as you give appropriate credit to the original author(s) and the source, provide a link to the Creative Commons licence, and indicate if changes were made. The images or other third party material in this article are included in the article's Creative Commons licence, unless indicated otherwise in a credit line to the material. If material is not included in the article's Creative Commons licence and your intended use is not permitted by statutory regulation or exceeds the permitted use, you will need to obtain permission directly from the copyright holder. To view a copy of this licence, visit http://creativecommons.org/licenses/by/4.0/.

\section{References}

1. Gerstein HC, Pogue J, Mann JFE, Lonn E, Dagenais GR, McQueen M et al (2005) The relationship between dysglycaemia and cardiovascular and renal risk in diabetic and non-diabetic participants in the HOPE study: a prospective epidemiological analysis. Diabetologia 48:1749-1755

2. Punthakee Z, Werstuck GH, Gerstein HC (2007) Diabetes and cardiovascular disease: explaining the relationship. Rev Cardiovasc Med 8:145-153

3. Xu Y, Bi Y, Li M, Wang T, Sun K, Xu M et al (2013) Significant coronary stenosis in asymptomatic Chinese with different glycemic status. Diabetes Care 36:1687-1694

4. Ji L, Hu D, Pan C, Weng J, Huo Y, Ma C et al (2013) Primacy of the $3 \mathrm{~B}$ approach to control risk factors for cardiovascular disease in type 2 diabetes patients. Am J Med 126(925):e11-e22

5. Ray KK, Seshasai SR, Wijesuriya S, Sivakumaran R, Nethercott $S$, Preiss D et al (2009) Effect of intensive control of glucose on cardiovascular outcomes and death in patients with diabetes mellitus: a meta-analysis of randomised controlled trials. Lancet 373:1765-1772

6. Gaede P, Lund-Andersen H, Parving HH, Pedersen O (2008) Effect of a multifactorial intervention on mortality in type 2 diabetes. N Engl J Med 358:580-591

7. Kuhl J, Jorneskog G, Wemminger M, Bengtsson M, Lundman P, Kalani M (2015) Long-term clinical outcome in patients with acute coronary syndrome and dysglycaemia. Cardiovasc Diabetol $14: 120$

8. Thygesen K, Alpert JS (2000) Myocardial infarction redefined-a consensus document of The Joint European Society of Cardiology/American College of Cardiology Committee for the redefinition of myocardial infarction. Eur Heart J 21:1502-1513
9. Virmani R, Burke AP, Kolodgie F (2006) Morphological characteristics of coronary atherosclerosis in diabetes mellitus. Can J Cardiol 22(Suppl B):81B-84B

10. Burchfield JS, Xie M, Hill JA (2013) Pathological ventricular remodeling: mechanisms: part 1 of 2. Circulation 128:388-400

11. Fox CS, Golden SH, Anderson C, Bray GA, Burke LE, de Boer IH et al (2015) Update on prevention of cardiovascular disease in adults with type 2 diabetes mellitus in light of recent evidence: a scientific statement from the American Heart Association and the American Diabetes Association. Diabetes Care 38:1777-1803

12. Kucharska-Newton A, Griswold M, Yao ZH, Foraker R, Rose K, Rosamond W et al (2017) Cardiovascular disease and patterns of change in functional status over 15 years: Findings from the Atherosclerosis Risk in Communities (ARIC) Study. J Am Heart Assoc 6:pii:e004144

13. Herrington W, Lacey B, Sherliker P, Armitage J, Lewington S (2016) Epidemiology of atherosclerosis and the potential to reduce the global burden of atherothrombotic disease. Circ Res 118:535-546

14. Barquera S, Pedroza-Tobías A, Medina C, Hernández-Barrera L, Bibbins-Domingo K, Lozano R et al (2015) Global overview of the epidemiology of atherosclerotic cardiovascular disease. Arch Med Res 46:328-338

15. Libby P (2016) Assisted living in the atheroma: elderly macrophages promote plaques. Cell Metab 24:779-781

16. Shimizu I, Yoshida Y, Katsuno T, Tateno K, Okada S, Moriya J et al (2012) p53-induced adipose tissue inflammation is critically involved in the development of insulin resistance in heart failure. Cell Metab 15:51-64

17. ACCORD Study Group, Cushman WC, Evans GW, Byington RP, Goff DC Jr, Grimm RH Jr et al (2010) Effects of intensive blood-pressure control in type 2 diabetes mellitus. N Engl J Med. 362:1575-1585

18. Li N, Katzmarzyk PT, Horswell R, Zhang Y, Li W, Zhao W et al (2014) BMI and coronary heart disease risk among low-income and underinsured diabetic patients. Diabetes Care 37:3204-3212

19. Kuo JF, Hsieh YT, Mao IC, Lin SD, Tu ST, Hsieh MC (2015) The association between body mass index and all-cause mortality in patients with type 2 diabetes mellitus: a 5.5-year prospective analysis. Medicine (Baltimore) 2015(94):e1398

20. Braga F, Pasqualetti S, Ferraro S, Panteghini M (2016) Hyperuricemia as risk factor for coronary heart disease incidence and mortality in the general population: a systematic review and metaanalysis. Clin Chem Lab Med 54:7-15

21. Zuo T, Liu X, Jiang L, Mao S, Yin X, Guo L (2016) Hyperuricemia and coronary heart disease mortality: a meta-analysis of prospective cohort studies. BMC Cardiovasc Disord 16:207

22. Li M, Hu X, Fan Y, Li K, Zhang X, Hou W et al (2016) Hyperuricemia and the risk for coronary heart disease morbidity and mortality a systematic review and dose-response meta-analysis. Sci Rep 6:19520

23. Wu J, Qiu L, Cheng XQ, Xu T, Wu W, Zeng XJ et al (2017) Hyperuricemia and clustering of cardiovascular risk factors in the Chinese adult population. Sci Rep 7:5456

24. Zhang JW, He LJ, Cao SJ, Yang Q, Yang SW, Zhou YJ (2014) Association of serum uric acid and coronary artery disease in premenopausal women. PLoS ONE 9:e106130

25. Rosenson RS, Brewer HB, Rader DJ (2014) Lipoproteins as biomarkers and therapeutic targets in the setting of acute coronary syndrome. Circ Res 114:1880-1889

26. Action to Control Cardiovascular Risk in Diabetes Study Group, Gerstein HC, Miller ME, Byington RP, Goff DC Jr, Bigger JT et al (2008) Effects of intensive glucose lowering in type 2 diabetes. $\mathrm{N}$ Engl J Med 358:2545-2559

27. ADVANCE Collaborative Group, Patel A, MacMahon S, Chalmers J, Neal B, Billot L et al (2008) Intensive blood glucose control 
and vascular outcomes in patients with type 2 diabetes. $\mathrm{N}$ Engl $\mathrm{J}$ Med 358:2560-2572

28. Duckworth W, Abraira C, Moritz T, Reda D, Emanuele N, Reaven PD et al (2009) Glucose control and vascular complications in veterans with type 2 diabetes. N Engl J Med 360:129-139

29. Holman RR, Paul SK, Bethel MA, Matthews DR, Neil HA (2008) 10-year follow-up of intensive glucose control in type 2 diabetes. N Engl J Med 359:1577-1589

30. Blomster JI, Zoungas S, Chalmers J, Li Q, Chow CK, Woodward $\mathrm{M}$ et al (2014) The relationship between alcohol consumption and vascular complications and mortality in individuals with type 2 diabetes. Diabetes Care 37:1353-1359

31. Messerli FH, Mancia G, Conti CR, Hewkin AC, Kupfer S, Champion A et al (2006) Dogma disputed: can aggressively lowering blood pressure in hypertensive patients with coronary artery disease be dangerous? Ann Intern Med 144:884-893

32. Protogerou AD, Safar ME, Iaria P, Safar H, Le Dudal K, Filipovsky J et al (2007) Diastolic blood pressure and mortality in the elderly with cardiovascular disease. Hypertension 50:172-180

33. Bangalore S, Qin J, Sloan S, Murphy SA, Cannon CP (2010) What is the optimal blood pressure in patients after acute coronary syndromes? Relationship of blood pressure and cardiovascular events in the PRavastatin OR atorVastatin Evaluation and Infection Therapy-Thrombolysis In Myocardial Infarction (PROVE IT-TIMI) 22 trial. Circulation 122:2142-2151

34. Nordestgaard BG, Chapman MJ, Ray K, Borén J, Andreotti F, Watts GF et al (2010) Lipoprotein(a) as a cardiovascular risk factor: current status. Eur Heart J 31:2844-2853

35. Stone NJ, Robinson JG, Lichtenstein AH, Bairey Merz CN, Blum CB, Eckel RH et al (2014) 2013 ACC/AHA guideline on the treatment of blood cholesterol to reduce atherosclerotic cardiovascular risk in adults: a report of the American College of Cardiology/ American Heart Association Task Force on Practice Guidelines. Circulation 129(25 Suppl 2):S1-S45

36. Emerging Risk Factors Collaboration, Erqou S, Kaptoge S, Perry PL, Di Angelantonio E, Thompson A et al (2009) Lipoprotein(a) concentration and the risk of coronary heart disease, stroke, and nonvascular mortality. JAMA 302:412-423
37. Kamstrup PR, Benn M, Tybjærg-Hansen A, Nordestgaard BG (2008) Extreme lipoprotein(a) levels and risk of myocardial infarction in the general population: the Copenhagen City Heart Study. Circulation 117:176-184

38. Kamstrup PR, Tybjaerg-Hansen A, Steffensen R, Nordestgaard BG (2009) Genetically elevated lipoprotein(a) and increased risk of myocardial infarction. JAMA 301:2331-2339

39. Clarke R, Peden JF, Hopewell JC, Kyriakou T, Goel A, Heath SC et al (2009) Genetic variants associated with $L p(a)$ lipoprotein level and coronary disease. N Engl J Med 361:2518-2528

40. Perak AM, Ning H, de Ferranti SD, Gooding HC, Wilkins JT, Lloyd-Jones DM (2016) Long-term risk of atherosclerotic cardiovascular disease in US adults with the familial hypercholesterolemia phenotype. Circulation 134:9-19

41. Alonso R, Andres E, Mata N, Fuentes-Jiménez F, Badimón L, López-Miranda J et al (2014) Lipoprotein(a) levels in familial hypercholesterolemia: an important predictor of cardiovascular disease independent of the type of LDL receptor mutation. J Am Coll Cardiol 63:1982-1989

42. Chan DC, Pang J, Hooper AJ, Burnett JR, Bell DA, Bates TR et al (2015) Elevated lipoprotein(a), hypertension and renal insufficiency as predictors of coronary artery disease in patients with genetically confirmed heterozygous familial hypercholesterolemia. Int J Cardiol 201:633-638

43. Ference BA, Bhatt DL, Catapano AL, Packard CJ, Graham I, Kaptoge $S$ et al (2019) Association of genetic variants related to combined exposure to lower low-density lipoproteins and lower systolic blood pressure with lifetime risk of cardiovascular disease. JAMA. https://doi.org/10.1001/jama.2019.14120

44. Larsen LE, Stoekenbroek RM, Kastelein JJP, Holleboom AG (2019) Moving targets: recent advances in lipid-lowering therapies. Arterioscler Thromb Vasc Biol 39:349-359

Publisher's Note Springer Nature remains neutral with regard to jurisdictional claims in published maps and institutional affiliations. 